\title{
ADSORÇÃO DE OLIGONUCLEOTÍDEOS EM NANOEMULSÕES OBTIDAS POR EMULSIFICAÇÃO ESPONTÂNEA
}

\author{
Érico Martini, Edison Carvalho e Helder Teixeira* \\ Faculdade de Farmácia, Universidade Federal do Rio Grande do Sul, Av. Ipiranga, 2752, 90610-000 Porto Alegre - RS, Brasil \\ Fabiana Leão e Mônica Cristina de Oliveira \\ Faculdade de Farmácia, Universidade Federal de Minas Gerais, Av. Presidente Antônio Carlos, 6627, 31270-901 Belo Horizonte \\ - MG, Brasil
}

Recebido em 21/7/06; aceito em 12/9/06; publicado na web em 27/4/07

\begin{abstract}
OLIGONUCLEOTIDE ADSORPTION ON NANOEMULSIONS OBTAINED BY SPONTANEOUS EMULSIFICATION. This paper describes the adsorption of an oligothymidylate $\left(\mathrm{pdT}_{16}\right)$ on nanoemulsions obtained by spontaneous emulsification procedures. Formulations were composed by medium chain triglycerides, egg lecithin, glycerol, water (NE) and stearylamine (NE $\mathrm{SA}_{\mathrm{A}}$ ). After optimization of operating conditions, the mean droplet size was smaller than $255 \mathrm{~nm}$. Adsorption isotherms showed a higher amount of $\mathrm{pdT}_{16}$ adsorbed on cationic $\mathrm{NE}_{\mathrm{SA}}(60 \mathrm{mg} / \mathrm{g})$ compared to $\mathrm{NE}(20 \mathrm{mg} / \mathrm{g})$. pdT ${ }_{16}$ adsorption was also evidenced by the inversion of the $\zeta$-potential of $\mathrm{NE}_{\mathrm{SA}}$ (from +50 to $-30 \mathrm{mV}$ ) and the morphology of oil droplets examined through transmission electron microscopy. The overall results showed the role of electrostatic interactions on the adsorption of $\mathrm{pdT}_{16}$ on the oil/water interface of nanoemulsions.
\end{abstract}

Keywords: cationic nanoemulsions; oligonucleotides; adsorption.

\section{INTRODUÇÃO}

A complexação de polinucleotídeos em sistemas coloidais lipídicos catiônicos (i.e., lipossomas, nanopartículas e nanoemulsões) tem sido considerada como uma estratégia promissora para sua administração in vivo ${ }^{1-4}$.

Nos últimos anos, a formação de complexos entre polinucleotídeos e nanoemulsões catiônicas tem sido particularmente descrita na literatura ${ }^{5-14}$. Nanoemulsões catiônicas são geralmente constituídas de um núcleo oleoso (de origem natural ou semi-sintética) estabilizado por mistura binária de fosfolipídeos e lipídeos catiônicos. Os polinucleotídeos formam espontaneamente um complexo em meio aquoso através de interações eletrostáticas entre os grupamentos fosfato e os grupamentos carregados positivamente dos lipídeos catiônicos. A carga de superfície positiva dessas nanoestruturas conduz a um aumento significativo da penetração intracelular dos polinucleotídeos, em decorrência das interações com as membranas celulares carregadas negativamente ${ }^{8,10,14}$. Além disso, a formação do complexo reduz a cinética de degradação dos polinucleotídeos frente ao ataque enzimático ${ }^{6,7,11,12}$.

Estudos relativos à formação de complexos entre polinucleotídeos em fita simples, denominados oligonucleotídeos (ON), e nanoemulsões catiônicas obtidas por microfluidização foram descritos por Teixeira e colaboradores ${ }^{11-14}$. A carga de superfície e o tamanho do fragmento foram identificados como os principais fatores que influenciam a eficiência de complexação do $\mathrm{ON}$ com a nanoestrutura ${ }^{11}$. Estudos posteriores de potencial zeta, transferência de energia de fluorescência e de tensão interfacial permitiram caracterizar a interação dos oligonucleotídeos com os constituintes das formulações (fosfolipídeos e/ou lipídeos catiônicos), sugerindo a adsorção dos ON na interface óleo/água (O/A) das nanoemulsões ${ }^{12,13}$.

Recentemente, foi descrita na literatura ${ }^{15,16}$ a viabilidade de preparação de nanoemulsões catiônicas contendo ON, através do procedimento de emulsificação espontânea. O interesse desse procedi-

*e-mail: helder@farmacia.ufrgs.br mento reside, entre outros, na sua adequação para preparação de pequenos lotes de formulação, a temperaturas moderadas, em estudos de bancada e na possibilidade de funcionalização da interface das nanoemulsões, através da nanodeposição de polímeros pré-for$\operatorname{mados}^{15,17}$. Esse procedimento baseia-se na emulsificação espontânea dos constituintes da fase interna (previamente solubilizados em solvente orgânico) em água, seguida da retirada do solvente geralmente por destilação sob pressão reduzida. A formação das gotículas ocorre quando a fase orgânica é vertida na fase aquosa, provocando uma turbulência interfacial que ocorre durante a difusão do solvente orgânico na água, observando-se rápido espalhamento da interface, como resultado da difusão mútua entre os solventes, o que fornece energia suficiente para formação das gotículas ${ }^{18}$.

Diversos parâmetros podem influenciar as propriedades físicoquímicas das nanoemulsões obtidas, entre eles, as condições de emulsificação empregadas e a composição quali e quantitativa das formulações ${ }^{18,19}$. Como um exemplo recente, Bouchemal e colaboradores $^{18}$ descreveram o efeito da viscosidade e do equilíbrio hidrófilo-lipófilo do núcleo oleoso e a miscibilidade da fase orgânica em água sobre o diâmetro de gotícula das nanoemulsões.

Neste estudo, a primeira etapa teve por objetivo avaliar a influência da natureza e quantidade de solvente orgânico utilizado no procedimento de emulsificação espontânea sobre o diâmetro de gotícula das nanoemulsões. Após essa etapa de otimização, a isoterma de adsorção de um oligonucleotídeo politimidilato ( $\mathrm{pdT}_{16}$ ) foi realizada, bem como a avaliação da influência da adsorção do ON sobre a morfologia e as propriedades físico-químicas das nanoemulsões (i.e., diâmetro, potencial zeta e viscosidade).

\section{PARTE EXPERIMENTAL}

\section{Materiais}

$\operatorname{pdT}_{16}$ e estearilamina (SA) foram adquiridos junto à Invitrogen (Brasil) e Sigma (EUA), respectivamente. Triglicerídeos de cadeia média dos ácidos cáprico e caprílico (TCM) e lecitina de gema de 
ovo (Lipoid E-80 ${ }^{\circledR}$ ) foram gentilmente doados pela Lipoid $\mathrm{GmbH}$ (Alemanha). Todos os outros reagentes e solventes utilizados foram de grau analítico.

\section{Preparação das nanoemulsões}

As nanoemulsões foram preparadas através do procedimento de emulsificação espontânea. Os constituintes das fases oleosa e aquosa foram solubilizados em solvente orgânico e água, respectivamente. Em seguida, a fase orgânica foi vertida sobre a fase aquosa através de funil calibrado, sob agitação moderada e constante, seguida da retirada do solvente através de evaporação sob pressão reduzida em evaporador rotatório. A Tabela 1 apresenta a composição final das formulações. A fim de otimizar as condições de emulsificação espontânea, as nanoemulsões foram preparadas com quantidades crescentes de acetona/água e etanol/água, nas proporções de $1: 2 ; 2: 4 ; 3: 6$ e 4:8 (solvente orgânico:água) para a obtenção de $1 \mathrm{~mL}$ de formulação final. Após o preparo, o pH das formulações foi ajustado a cerca de 7 .

Tabela 1. Composição final das nanoemulsões $\mathrm{NE}$ e $\mathrm{NE}_{\mathrm{SA}}(\%, \mathrm{~m} / \mathrm{m})$

\begin{tabular}{lcr}
\hline & $\mathrm{NE}$ & $\mathrm{NE}_{\mathrm{SA}}$ \\
\hline Lipoid E-80 & 2,00 & 2,00 \\
TCM & 8,00 & 8,00 \\
Estearilamina & - & 0,05 \\
Glicerol & 2,25 & 2,25 \\
Água q.s.p & 100,00 & 100,00 \\
\hline
\end{tabular}

\section{Validação da metodologia para quantificação do $\mathbf{p d T} \mathbf{T}_{16}$}

Os parâmetros de validação selecionados para doseamento do $\mathrm{pdT}_{16}$ por UV seguiram as normas de validação dos procedimentos analíticos e de metodologias do "International Conference on the Harmonization of Technical Requirements for the Registration of Pharmaceuticals for Human Use" ${ }^{20}$. Foram avaliados os parâmetros de linearidade, repetibilidade, precisão intermediária e especificidade. Os ensaios foram realizados em um comprimento de onda de $266 \mathrm{~nm}$, utilizando-se espectrofotômetro UV-Visível modelo Hewlett-Packard 8452A. O método mostrou-se linear $\left(r^{2}=0,9974\right)$ em uma gama de concentração de 2,5 a $25 \mu \mathrm{g} / \mathrm{mL}$ e preciso (DPR = 2,05 e $1,70 \%$, para repetibilidade e precisão intermediária, respectivamente). A especificidade foi testada na presença de glicerol (fase externa das nanoemulsões), demonstrando que esse adjuvante não influencia na quantificação do $\operatorname{pdT}_{16}$.

\section{Estudos de adsorção do $\mathbf{p d T}_{16}$}

Em uma primeira etapa, a recuperação do $\mathrm{pdT}_{16}$ foi determinada em unidades de ultrafiltração constituídas de celulose regenerada de $30 \mathrm{kDa}$ (Ultrafree ${ }^{\circledR}$, Millipore ${ }^{\circledR}$ ). Soluções aquosas contendo pdT ${ }_{16}$ (25 a $250 \mu \mathrm{g} / \mathrm{mL}$ ) foram centrifugadas e a quantidade de $\mathrm{pdT}_{16}$ no ultrafiltrado foi determinada. Por diferença entre a quantidade inicial e a detectada foi determinada a taxa de recuperação (\%) do $\operatorname{pdT}_{16}$.

Para os estudos de adsorção, soluções aquosas contendo o pdT 16 ( $25 \mathrm{a} 250 \mu \mathrm{g} / \mathrm{mL}$ ) foram preparadas. As nanoemulsões ( $\mathrm{NE}$ e $\mathrm{NE}_{\mathrm{SA}}$ ) foram então adicionadas às soluções aquosas de $\mathrm{pdT}_{16}$ à temperatura ambiente. Após $30 \mathrm{~min}$, as amostras foram colocadas nas unidades de ultrafiltração e centrifugadas a uma velocidade de 5.000 rpm por 20 min e a concentração de pdT $_{16}$ não adsorvida foi determinada no ultrafiltrado. Para todas as amostras foi realizado um espectro de absorção no UV na faixa de 200 a $400 \mathrm{~nm}$, a fim de verificar a ausência de qualquer pico interferente. Em todos os casos, a concentração final de fase interna das nanoemulsões no meio de adsorção foi de $2 \mathrm{mg} / \mathrm{mL}$. Os resultados foram expressos como a média de três experimentos independentes e a quantidade de $\mathrm{pdT}_{16}$ adsorvida por grama de fase interna de nanoemulsão foi obtida através da Equação

$Q_{a}=\frac{q_{i}-q_{r}}{q_{n e}}$

onde, $Q_{a}=$ quantidade de $\mathrm{pdT}_{16}$ adsorvido $\left(\mathrm{mg}\right.$ de $\mathrm{pdT}_{16} / \mathrm{g}$ de fase inter$\mathrm{na}) ; q_{i}=$ quantidade de $\mathrm{pdT}_{16}$ inicial adicionada às nanoemulsões $(\mathrm{mg})$; $q_{r}=$ quantidade de $\mathrm{pdT}_{16}$ recuperada no ultrafiltrado $(\mathrm{mg}) \mathrm{e}, q_{n e}=$ quantidade de fase interna de nanoemulsão no meio de adsorção $(\mathrm{g})$.

\section{Caracterização das nanoemulsões}

As nanoemulsões foram caracterizadas antes e após a adsorção do $\mathrm{pdT}_{16}$, como segue: o diâmetro médio das gotículas e o potencial zeta das formulações foram determinados através de espectroscopia de correlação de fótons e da mobilidade eletroforética, respectivamente, utilizando-se um Zetasizer 3000HS (Malvern Instruments Ltd., Reino Unido), após adequada diluição das amostras. $\mathrm{O}$ pH das nanoemulsões foi determinado utilizando-se potenciômetro digital Micronal B 474 (Micronal, São Paulo, Brasil), antes e após o seu ajuste a cerca de 7 . A viscosidade das nanoemulsões foi determinada por viscosimetria capilar, segundo a Farmacopéia Brasileira ${ }^{21}$. Após a determinação do tempo de escoamento, calculou-se a viscosidade das nanoemulsões usando a densidade determinada em picnômetro. A morfologia das gotículas de nanoemulsões foi avaliada por microscopia eletrônica de transmissão (MET). As amostras foram adicionadas em suportes metálicos (200 mesh) de cobre com revestimento de carbono e Formvar, sendo o contraste negativo realizado com acetato de uranila a $2 \%$ $(\mathrm{m} / \mathrm{v})$. Em seguida, as amostras foram armazenadas por $24 \mathrm{~h} \mathrm{e}$, então, submetidas à análise através de MET, com aumento de 100.000 vezes.

\section{Análise estatística}

Os resultados foram analisados através de teste " $\mathrm{t}$ " de Student, considerando-se um nível de significância de 0,05.

\section{RESULTADOS E DISCUSSÃO}

A Figura 1 apresenta a influência da natureza e do volume dos solventes empregados no procedimento de emulsificação espontânea sobre o diâmetro de gotícula e índice de polidispersidade das nanoemulsões $\mathrm{NE} \mathrm{e} \mathrm{NE}_{\mathrm{SA}}$. Etanol e acetona foram selecionados para o procedimento de emulsificação espontânea, uma vez que são solventes miscíveis com água e classificados como de baixo potencial tóxico ${ }^{22}$. Como pode ser observado, independente da natureza do solvente empregado e da presença do lipídeo catiônico estearilamina, ocorre uma redução do diâmetro de gotícula das nanoemulsões, até cerca de 250 $\mathrm{nm}$, com o aumento progressivo da quantidade de solvente utilizada. A redução do diâmetro das gotículas pode ser atribuída à maior velocidade de difusão da fase orgânica em água, como consequiência da menor concentração dos componentes da fase interna solubilizados na solução orgânica ${ }^{18,23}$. Contudo, não foi observada uma redução suplementar do diâmetro de gotícula $(\mathrm{p}<0,05)$ para a maior quantidade de solventes empregada (proporção 4/8), sugerindo que, a partir de uma determinada concentração do soluto, a velocidade de difusão do solvente orgânico não é mais influenciada pela mesma, mas apenas pelas propriedades de difusão do solvente empregado. 

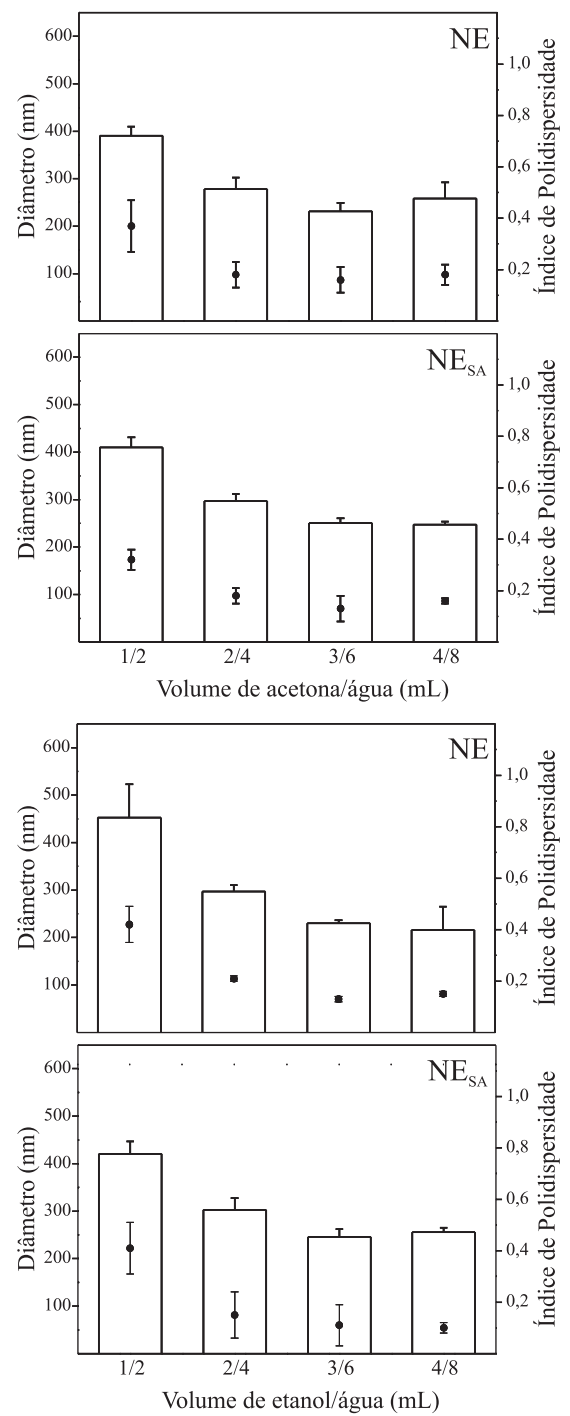

Figura 1. Diâmetro médio de gotícula (barras) e índice de polidispersidade (símbolos) da nanoemulsão isenta de lipídeo catiônico (NE) ou contendo o lipídeo catiônico estearilamina $\left(N E_{S A}\right)$, utilizando acetona ou etanol como solvente no procedimento de emulsificação espontânea

A NE isenta de lipídeo catiônico apresenta um potencial zeta negativo (Tabela 2) devido à presença de fosfolipídeos ácidos na lecitina de gema de ovo, como a fosfatidilserina e o ácido fosfatídico ${ }^{24}$. A adição de estearilamina à nanoemulsão conduz à inversão do potencial zeta para valores positivos, demonstrando sua localização na interface O/A. A estearilamina é um lipídeo catiônico que apresenta um $\mathrm{pK}$ de 10,6 , conferindo assim uma carga global positiva no $\mathrm{pH}$ final das nanoemulsões. A presença do lipídeo catiônico também influencia o $\mathrm{pH}$ final da formulação $\mathrm{NE}_{\mathrm{SA}}$, indicando o efeito do seu grupamento polar, amina primária, sobre esse parâmetro.

Tabela 2. Propriedades físico-químicas das nanoemulsões $\mathrm{NE}$ e $\mathrm{NE}_{\mathrm{SA}}$

\begin{tabular}{lcc}
\hline & $\mathrm{NE}$ & $\mathrm{NE}_{\mathrm{SA}}$ \\
\hline $\mathrm{pH}^{\mathrm{a}}$ & $6,36 \pm 0,10$ & $8,70 \pm 0,02$ \\
Diâmetro de gotícula $(\mathrm{nm})$ & $230 \pm 7$ & $255 \pm 14$ \\
Potencial zeta $(\mathrm{mV})$ & $-32 \pm 2$ & $50 \pm 8$ \\
Viscosidade $(\mathrm{cP})$ & $1,50 \pm 0,02$ & $1,60 \pm 0,03$ \\
\hline
\end{tabular}

( ${ }^{\mathrm{a}} \mathrm{pH}$ das nanoemulsões antes do ajuste a cerca de 7 .
A avaliação da adsorção de ON com nanoemulsões tem sido realizada de maneira indireta ${ }^{11-13}$. De fato, o ON não complexado é quantificado em uma fração da fase aquosa externa das nanoemulsões, obtida após prévia separação utilizando-se membranas de ultrafiltração. A determinação da recuperação do $\mathrm{ON}$ através das membranas representa uma etapa prévia fundamental para se avaliar se a membrana não representa um fator limitante à passagem do ON solúvel na fase aquosa externa. Neste estudo, a taxa de recuperação do $\operatorname{pdT}_{16}$ em solução aquosa foi superior a $98 \%$, na faixa de concentração das isotermas de adsorção, comprovando que a membrana de ultrafiltração selecionada não representa um fator limitante para a passagem do $\mathrm{ON}$ pdT ${ }_{16}$ solúvel na fase aquosa externa.

A Figura 2 apresenta as isotermas de adsorção do pdT ${ }_{16}$ para as formulações $\mathrm{NE}$ e $\mathrm{NE}_{\mathrm{SA}}$. Pode-se observar a adsorção do pdT $\mathrm{T}_{16}$ até cerca de $20 \mathrm{mg} / \mathrm{g}$ de fase interna de NE. Essa reduzida adsorção deve estar relacionada com as propriedades de superfície dessas formulações. O potencial zeta negativo da formulação NE (Tabela 2) pode limitar o acesso dos $\mathrm{ON}$ à interface, devido a fenômenos de repulsão eletrostática ${ }^{13,17}$. Contudo, para $\mathrm{NE}_{\mathrm{SA}}$ a adsorção do $\mathrm{pdT}_{16}$ foi significativamente superior. A isoterma pode ser dividida em duas etapas. $\mathrm{Na}$ primeira, ocorre um aumento progressivo da quantidade de $\mathrm{pdT}_{16}$ adsorvido até um máximo ( $\sim 60 \mathrm{mg} / \mathrm{g}$ de fase interna). Possivelmente, isto ocorre devido à elevada afinidade das cargas positivas das gotículas pelos grupamentos fosfato carregados negativamente do ON. Esses resultados indicam que a presença do lipídeo catiônico na interface de $\mathrm{NE}_{\mathrm{SA}}$ aumenta substancialmente a capacidade de adsorção do $\mathrm{pdT}_{16}$, através de interações eletrostáticas. Na segunda etapa, quantidades suplementares de $\mathrm{pdT}_{16}$ não alteram a quantidade de $\mathrm{pdT}_{16}$ associado, sugerindo o alcance da saturação dos sítios de interação carregados positivamente para a associação dos $\mathrm{ON}$.

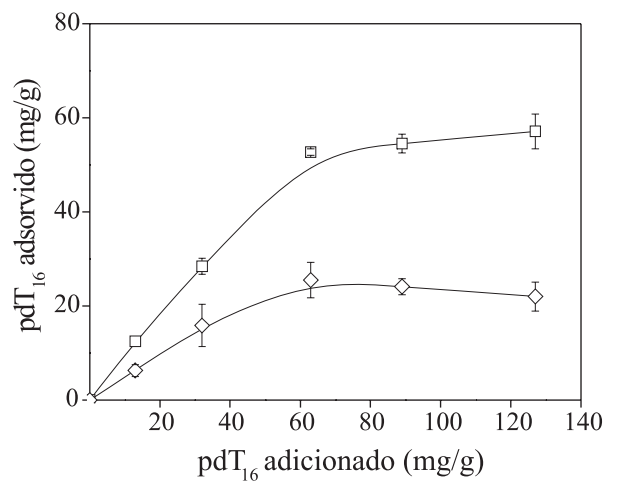

Figura 2. Quantidade de $O N$ pdT $T_{16}$ adsorvido às nanoemulsões $N E(\diamond)$ ou $N E_{S A}(\square)$ em função da quantidade de $p d T_{16}$ adicionado

A Figura 3 apresenta a influência da adsorção do pdT $_{16}$ nas propriedades físico-químicas da $\mathrm{NE}_{\mathrm{SA}}$. Como pode ser observado, existe uma tendência ao aumento do diâmetro de gotícula de $\mathrm{NE}_{\mathrm{SA}}(3 \mathrm{~A})$ com a adsorção do $\operatorname{pdT}_{16}$. $\mathrm{O}$ diâmetro médio encontra-se próximo a $300 \mathrm{~nm}$ sendo, em todos os casos, superior ao diâmetro original (Tabela 2), sugerindo que a adsorção do $\mathrm{pdT}_{16}$ conduz a um aumento do raio hidrodinâmico das gotículas. No caso específico das concentrações compreendidas entre cerca de 3 e $30 \mathrm{mg} / \mathrm{g}$, o diâmetro médio é superior a $300 \mathrm{~nm}$. Esse resultado deve estar relacionado com a zona de inversão do potencial zeta de $\mathrm{NE}_{\mathrm{SA}}$, o que poderia no momento da adsorção do $\mathrm{pdT}_{16}(3 \mathrm{C})$ ter originado agregados resultantes da neutralização de cargas. Apesar das diferenças em relação ao diâmetro (3A), os resultados demonstram que não existe influência do $\operatorname{pdT}_{16}$ na viscosidade das nanoemulsões (3B): a viscosidade foi próxima de $1 \mathrm{cP}$, independente da concentração de $\mathrm{pdT}_{16}$. Contudo, observase uma redução de cerca de $60 \%$ na viscosidade em comparação aos 

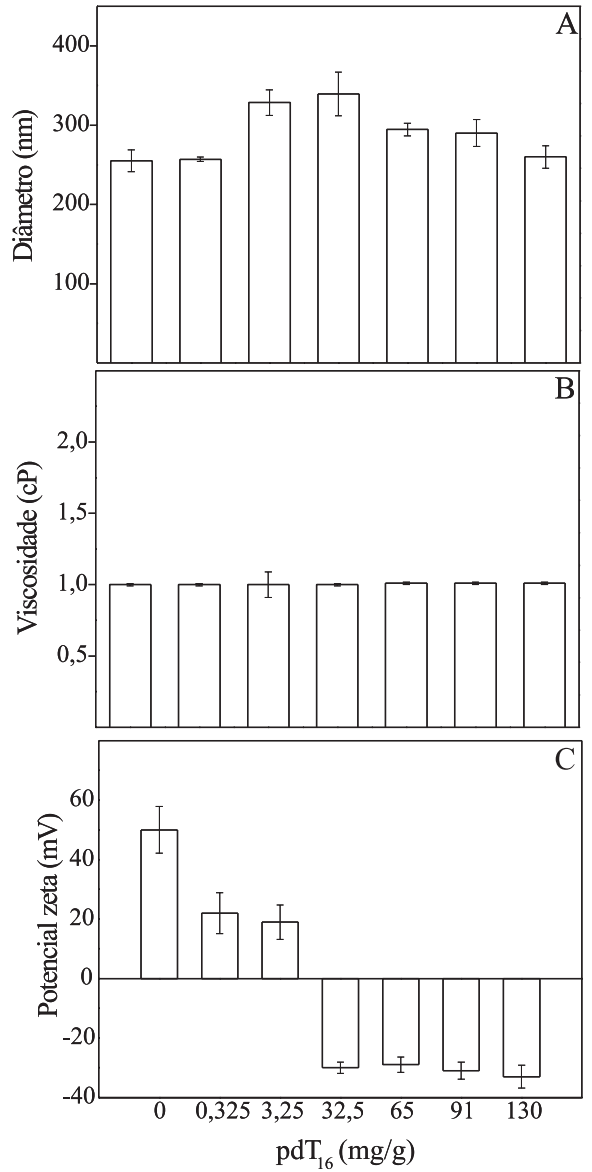

Figura 3. Diâmetro (A), viscosidade (B) e potencial zeta $(C)$ da nanoemulsão $N E_{S A}$, após adição de quantidades crescentes do pdT 16

valores obtidos antes da associação do $\mathrm{ON}$ (Tabela 2), indicando a influência da concentração final de fase interna $(2 \mathrm{mg} / \mathrm{mL})$, obtida após a adição da solução de ON. Uma menor concentração de fase interna reduz as interações interparticulares, podendo resultar em menor viscosidade das nanoemulsões ${ }^{25,26}$.

Em relação ao potencial zeta, a adição de quantidades crescentes de $\mathrm{pdT}_{16}$ conduz à sua redução (Figura $3 \mathrm{C}$ ). $\mathrm{O}$ potencial zeta de $\mathrm{NE}_{\mathrm{SA}}$ apresenta inicialmente um valor positivo de $50 \mathrm{mV}$. Após a adição das menores quantidades de pdT ${ }_{16}(0,325$ e $3,25 \mathrm{mg} / \mathrm{g})$, observa-se uma redução no potencial zeta até cerca de $20-30 \mathrm{mV}$. As concentrações maiores de $\mathrm{pdT}_{16}$ conduzem rapidamente a uma inversão do potencial zeta original para valores negativos de cerca de $-30 \mathrm{mV}$, indicando a neutralização das cargas positivas dos lipídeos catiônicos na interface da $\mathrm{NE}_{\mathrm{SA}}$. De fato, nas condições experimentais utilizadas, a relação de cargas [+/-] varia de 20 até 0,01 , portanto, justificando os resultados de potencial zeta observados.

A avaliação da morfologia foi realizada por MET antes e após associação do pdT $\mathrm{T}_{16}$ com NE e $\mathrm{NE}_{\mathrm{SA}}$ (Figura 4). As fotomicrografias (A e B) revelam estruturas esféricas de diâmetro na faixa de 200$300 \mathrm{~nm}$, com interface definida e sem influência da composição das nanoemulsões. Os resultados confirmam aqueles obtidos pela técnica de espectroscopia a laser de autocorrelação, como demonstrado na Tabela 2. As fotomicrografias C e D demonstram que os diâmetros de gotículas não sofrem influência marcante após adição do $\mathrm{pdT}_{16}$, sendo próximos aos valores observados antes da associação do pdT $_{16}$. Em contrapartida, os resultados sugerem a formação de uma camada mais espessa no limite das gotículas oleosas da $\mathrm{NE}_{\mathrm{SA}}$, indicando a presença do $\mathrm{pdT}_{16}$ associado à interface, corroborando os resultados obtidos na adsorção do $\operatorname{pdT}_{16}$ (Figura

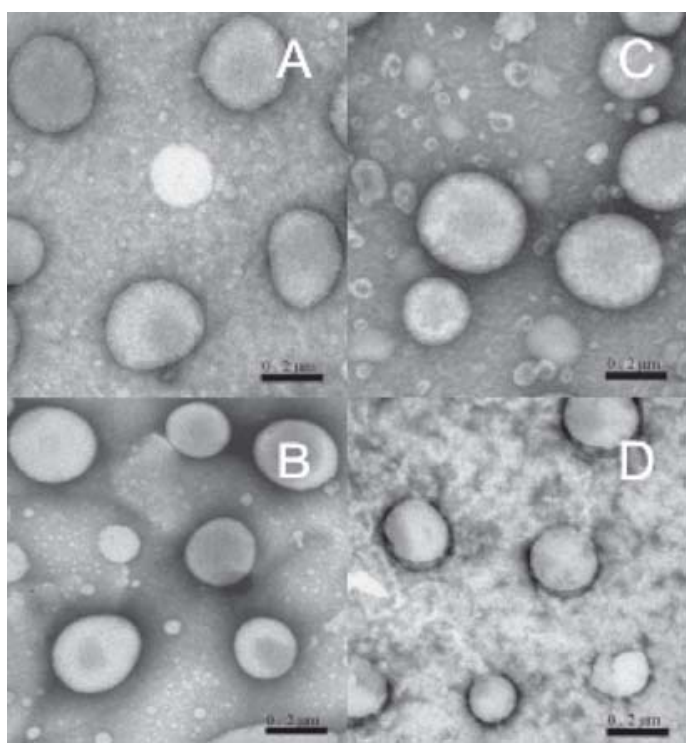

Figura 4. Fotomicrografias (MET) das nanoemulsões antes e após a associação do $p d T_{16}$ Legenda: $N E(A), N E / p d T_{16}(C), N E_{S A}(B)$ e $N E_{S A} / p d T_{16}(D)$

2). Assim, as áreas mais escuras observadas na interface das gotículas podem ser atribuídas à elevada afinidade do reagente de contraste (acetato de uranila) pelos ácidos nucléicos ${ }^{27,28}$.

\section{CONCLUSÕES}

Este estudo demonstrou, na primeira etapa, a possibilidade de modulação do diâmetro de gotícula das nanoemulsões a partir da otimização do volume de solventes empregados no procedimento de emulsificação espontânea. A adsorção do pdT $_{16}$ nas nanoemulsões foi claramente influenciada pela presença do lipídeo catiônico estearilamina na interface O/A. A maior associação do $\operatorname{pdT}_{16}$ à interface das $\mathrm{NE}_{\mathrm{SA}}$ é resultante das interações eletrostáticas estabelecidas entre ambos. Essa adsorção foi igualmente evidenciada pela inversão do potencial zeta e através da avaliação da morfologia das gotículas da fase interna por MET. No seu conjunto, esse trabalho demonstra as potencialidades de nanoemulsões obtidas por emulsificação espontânea como um sistema carreador de ON.

\section{AGRADECIMENTOS}

Ao CNPq, FAPERGS, FAPEMIG e CAPES/COFECUB n ${ }^{\circ}$ 540/ 06 pelo suporte financeiro e ao Centro de Microscopia Eletrônica da UFRGS pelo suporte técnico nas análises de MET.

\section{REFERÊNCIAS}

1. Woodle, M. C.; Scaria, P.; Curr. Opin. Colloid Interface Sci. 2001, 6, 78.

2. Brown, M. D.; Schätzlein, A. G.; Uchegbu, I. F.; Int. J. Pharm. 2001, 229,

3. Fattal, E.; Dubernet, C.; Couvreur, P.; S.T.P Pharma Sci. 2001, 11, 31.

4. Tabatt, K.; Kneuer, C.; Sameti, M.; Olbrich, C.; Muller, R. H.; Lehr, C. M.; Bakowsky, U.; J. Controlled Release 2004, 18, 321.

5. Choi, B. Y.; Chung, J. W.; Park, J. H.; Kim, K. H.; Kim, Y. I.; Koh, Y. H.; Kwon, J. W.; Lee, K. H.; Choi, H. J.; Kim, T. W.; Chung, H.; Kwon, I. C.; Jeong, S. Y.; Korean J. Radiol. 2002, 3, 194.

6. Bivas-Benita, M.; Oudshoorn, M.; Romeijn, S.; van Meijgaarden, C.; Koerten, H.; van Der Meulen, H.; Lambert, G.; Ottenhoff, T.; Benita, S. Junginger, H.; Borchard, G.; J. Controlled Release 2004, 100, 145.

7. Yi, S. W.; Yune, T. Y.; Kim, T. W.; Chung, H.; Choi, Y. W.; Kwon, I. C.; Lee, E. B.; Jeong, S. Y.; Pharm. Res. 2000, 17, 314.

8. Kim, T. W.; Chung, H.; Kwon, I. C.; Sung, H. C.; Shin, B. C.; Jeong, S. Y.; Int. J. Pharm. 2005, 295, 35. 
9. Hung, C. -H.; Hwang, T. -L.; Chang, C. -C.; Fang, J. -Y.; Int. J. Pharm. 2005, 289, 197.

10. Min, K. A.; Lee, S. K.; Kim, C. -K.; Biomaterials 2005, 26, 1063.

11. Teixeira, H.; Dubernet, C.; Puisieux, F.; Benita, S.; Couvreur, P.; Pharm. Res. 1999, 16, 1 .

12. Teixeira, H.; Dubernet, C.; Rosilio, V.; Laigle, A.; Deverre, J. R.; Scherman, D.; Benita, S.; Couvreur, P.; J. Controlled Release 2001, 70, 243.

13. Teixeira, H.; Rosilio, V.; Laigle, A.; Lepault, J.; Erk, I; Scherman, D.; Benita, S.; Couvreur, P.; Dubernet, C.; Biophys. Chem. 2001, 92, 169.

14. Teixeira, H.; Dubernet, C.; Chacun, H.; Rabinovich, L.; Boutet, T.; Deverre, J. R.; Benita, S.; Couvreur, P.; J. Controlled Release 2002, 89, 473.

15. Trimaille, T.; Chaix, C.; Delair, T.; Pichot, C.; Teixeira, H.; Dubernet, C.; Couvreur, P.; Colloid Polym. Sci. 2001, 279, 784.

16. Trimaille, T.; Chaix, C.; Pichot, C.; Delair, T.; J. Colloid Interface Sci. 2003, $258,135$.

17. Silva, C.; Martini, E.; Tavares, G.; Silveira, T.; De Oliveira, M. C.; Teixeira, H.; Acta Farm. Bonaerense 2006, 25, 169

18. Bouchemal, K.; Briançon, S.; Perrier, E.; Fessi, H.; Int. J. Pharm. 2004, $280,241$.
19. Park, K. -M.; Lee, M. -K.; Hwang, K. -J.; Kim, C. -K.; Int. J. Pharm. 1999, $183,145$.

20. ICH - International Conference on Harmonization of Technical Requirements for Registration of Pharmaceuticals for Human Use. Harmonised Tripartide Guideline Em Validation of analytical procedures, Methodology. Topic Q2B, 1996. Comission of the European Communities. IFPMA, Switzerland, 1996.

21. Farmacopéia Brasileira $4^{\mathrm{a}}$ ed., Atheneu: São Paulo, 1988, Pt.1, cap. V.2.7.

22. British Pharmacopoeia, $3^{\text {rd }}$ ed., The Stationery Office: London, 1999. CD Version.

23. Wehrle, P.; Magenheim, B.; Benita, S.; Eur. J. Pharm. Biopharm. 1995, $41,19$.

24. Li, L. C.; Tian, Y. Em Encyclopedia of Pharmaceutical Technology; $2^{\text {nd }}$ ed.; James, S.; James, C. B., eds.; Marcel Dekker Inc.: New York, 2002.

25. Ishii, F.; Sasaki, I.; Ogata, H.; J. Pharm. Pharmacol. 1990, 42, 513.

26. Jumaa, M.; Muller, B.; Int. J. Pharm. 1998, 163, 81.

27. Tarahovski, T. S.; Khusainova, R. S.; Gorelov, A. V.; Nicolaeva, T. I.; Deev, A. A.; Dawson, A. K.; Ivanitsky, V. R.; FEBS Lett. 1996, 390, 133.

28. Chevaillier, P.; Exp. Cell Res. 2004, 58, 213. 